\title{
Horizon universality and anomalous conductivities
}

\author{
Umut Gürsoy ${ }^{a}$ and Javier Tarrío ${ }^{b}$ \\ ${ }^{a}$ Institute for Theoretical Physics and Spinoza Institute, Utrecht University, \\ 3508 TD Utrecht, The Netherlands \\ ${ }^{b}$ Departament de Física Fonamental and Institut de Ciències del Cosmos, \\ Universitat de Barcelona, Martí i Franquès 1, ES-08028, Barcelona, Spain \\ E-mail: u.gursoy@uu.nl, j.tarrio@ub.edu
}

ABSTRACT: We show that the value of chiral conductivities associated with anomalous transport is universal in a general class of strongly coupled quantum field theories that admit a gravitational holographic dual in the large $\mathrm{N}$ limit. Our result only applies to theories in the presence of external gauge fields with no dynamical gluon fields. On the gravity side the result follows from near horizon universality of the fluctuation equations, similar to the holographic calculation of the shear viscosity.

KeYwords: Duality in Gauge Field Theories, AdS-CFT Correspondence, Anomalies in Field and String Theories

ARXiv EPRINT: 1410.1306 


\section{Contents}

1 Introduction 1

2 Action and equations of motion 4

$\begin{array}{lll}3 & \text { Fluctuations } & 6\end{array}$

4 Chiral magnetic and separation effects $\quad 8$

$\begin{array}{llr}5 & \text { Discussion } & 9\end{array}$

\section{Introduction}

Anomalous transport in quantum field theories with chiral fermions has enjoyed a renewal of interest since the recent discovery of the Chiral Magnetic Effect (CME) [1, 2]. In short, the CME refers to generation of a macroscopic electric current as a result of the axial anomaly in the presence of an external magnetic field $\vec{B}$

$$
\vec{J}=\sigma_{\mathrm{VV}} \vec{B}
$$

Here the "chiral magnetic conductivity" $\sigma_{\mathrm{VV}}$ is proportional to the anomaly coefficients. The main motivation to study the CME in the context of particle physics stems from its possible realization in the Heavy Ion Collision experiments. Indeed, in an off-central collision of heavy ions at RHIC and LHC, huge magnetic fields are expected to be generated by the "spectator" ions that do not participate in the formation of the Quark-Gluon Plasma (QGP) [3-9]. Then one can theoretically demonstrate [1,2] that the chiral anomaly in QCD with electromagnetic and gluon contributions gives rise to the CME in the off-central heavy ion collisions.

Presence of such anomaly-induced electric and chiral currents in the QGP might have imprints on the spectra of charged hadrons observed in the heavy ion collisions. Such experimental evidence is still controversial at present $[10,11]$. It is important to note that anomalous transport are also observed [12] in the so called the Weyl semi-metals which are recently realized in the laboratory [13]. These semi-metals can be viewed as strongly coupled electron-hole plasmas in 3 spatial dimensions with the single-particle excitations being chiral fermions [14-16].

In this paper we address the question whether the anomalous conductivities receive radiative corrections. We address this question in the holographic setting as general as possible. The axial current is known to enjoy both electromagnetic and QCD quantum anomalies that lead to the anomaly equation,

$$
\partial_{\mu} J_{5}^{\mu}=a_{1} F^{A} \wedge F^{A}+a_{2} F^{V} \wedge F^{V}+a_{3} \operatorname{Tr} G \wedge G,
$$


where $F^{A, V}$ are the field strengths of background axial ${ }^{1}$ and vector gauge fields, $G$ is field strength of the gluon field and $a_{i}$ are anomaly coefficients that are well-known to be one-loop exact $[17,18]$.

In the absence of perpendicular external (axio-)electric and (axio-)magnetic fields the first two terms in (1.2) vanish. The last term in (1.2) then is the main source of anomaly induced chiral imbalance. One can then effectively take into account such anomaly generating glue transitions ${ }^{2}$ by introducing an axial chemical potential $\mu_{5}$.

Then, one can show by various different methods [1,2], that the presence of the electromagnetic anomaly $a_{2} \neq 0$ in the presence of an external magnetic field $\vec{B}$ leads to generation of an electric current as in (1.1), with

$$
\sigma_{\mathrm{VV}}=\frac{e^{2}}{2 \pi^{2}} \mu_{5}
$$

This result makes explicit use of the anomaly for a single chiral species $[17,18]$, and $\sigma_{\mathrm{VV}}$ is the chiral magnetic conductivity. There is a similar effect, namely generation of a chiral current $J^{5}$ in response to a magnetic field that is $J^{5}=\sigma_{\mathrm{AV}} B$. This effect is called the chiral separation effect.

A fundamentally important question in anomalous transport is whether the value of the chiral magnetic conductivity above (and similarly other anomalous conductivities) receives radiative corrections or not. There exist a variety of arguments in favor of - at least perturbative - non-renormalization [2, 22-29], mainly due to the fact that the anomaly coefficients are one-loop exact $[17,18]$. The situation however is subtle and to make a clear statement about non-renormalization one has to distinguish the two types of anomalies that we will call type I and type II [30, 31]. The former type refers to anomalies that vanish when the external fields are turned off (i.e. they are 't Hooft anomalies), such as the first and the second terms in (1.2), whereas the type II anomalies refer to mixed gauge-global anomalies such as the gluonic contribution in (1.2) whose presence does not depend on external fields. In the latter case, one does expect radiative corrections generically [30, 32, 33].

In the absence of type II anomalies, on the other hand, there exists both direct and indirect methods establishing non-renormalization. Firstly, one can show absence of perturbative corrections directly in field theory using the axial and vector Ward identities and some recently proven non-renormalization theorems [34], see for example [35]. Absence of non-perturbative renormalization can also be established by two indirect methods. Firstly, assuming that a hydrodynamic description of the system is at hand, demanding a positive definite divergence of the entropy current determines $\sigma_{\mathrm{VV}}$ to be exactly as in (1.3), as shown in [36] (see also [37]). Secondly, [38] established a Euclidean effective field theory for anomalous transport, whose consistency again requires fixing the value of $\sigma_{\mathrm{VV}}$ as in (1.3).

In this note we address the question of whether the value of anomalous conductivities such as $\sigma_{\mathrm{VV}}$ in (1.3) are exact in strongly coupled quantum field theories that admit a gravitational dual description a la AdS/CFT [39-41]. The study of anomalous transport via the holographic correspondence played a major role in the development of the subject

\footnotetext{
${ }^{1}$ Even though there is no background axial gauge fields in nature, here we include them for generality.

${ }^{2}$ In QCD at finite temperature the dominant process is the sphaleron decays [19-21].
} 
from early on [42, 43]. In holography one introduces anomalous currents by considering bulk gauge fields in the presence of bulk Chern-Simons terms [41]. One can then calculate the anomalous conductivities using the Kubo's formulae by calculating the retarded Green's functions following the standard prescription of the holographic correspondence. The case of conformal plasma of $\mathcal{N}=4$ super Yang-Mills in the large $\mathrm{N}$ limit was first considered in the holographic description, in a series of papers by Landsteiner et al. [44-47]. By comparison of the holographic and weak coupling results, these authors concluded that the chiral magnetic conductivity receives no corrections at all. However, this is a very special theory, and one is immediately prompted to analyze the situation in a more general class of theories, in particular theories with a mass gap and running gauge coupling. Such a study was undertaken very recently by one of the authors together with A. Jansen in [48]. In that paper the anomalous conductivities were calculated in a holographic setting that is dual to a non-conformal theory that exhibits a confinement-deconfinement transition. One finds that the value in (1.3) non-trivially depends on the parameters of the gravitational background, hence it seems that the universal value in (1.3) no longer holds. However, when the result is expressed in terms of physical quantities such as the chemical potential $\mu_{5}$ and temperature $T$, one again finds that the anomalous conductivities attain their universal values. In particular the chiral magnetic conductivity is again precisely given by (1.3) [48].

The non-trivial result obtained in [48] prompted us to seek for a generic, backgroundindependent mechanism to explain the universality of anomalous conductivities in the holographic setting. In a sense, in this paper we seek for the holographic analog of the nonrenormalization theorems in field theory, that we summarized above. Such universal values for the transport coefficients would typically result from the universal near-horizon behavior of bulk fluctuations in black-brane backgrounds. The most famous example of such behavior is the universal value of the shear viscosity to entropy density ratio $\eta / s=1 / 4 \pi$ in gravitational theories quadratic in derivatives $[49,50]$. This robust result can indeed be explained by the background-independence of metric fluctuations near the horizon, see for example [51].

In case of the anomalous conductivities, such direct proofs of universality prove difficult because, unlike the case of the shear viscosity or electric conductivity, calculation of anomalous conductivities in holography involves mixing of bulk gauge and metric fluctuations. In a way, in order to establish universality one has to diagonalize these fluctuations which turns out to be an onerous task. A simpler case was studied in [52], where the authors considered gravity theories with no scalars and looked at the holographic flow of the chiral condutivities, i.e. their dependence on the radial coordinate. Particularly, in the case of the AdS-Reissner-Nordström blackhole, they found no holographic flow except the scale dependence of the chemical potentials.

In this paper, we address the calculation in a general class of two-derivative gravity models in an alternative way, namely by including the source, i.e., the axial or the vector magnetic field, in the fluctuations themselves. This method was already introduced by Donos and Gauntlett in a different context where the authors study the thermoelectric properties of holographic plasmas [53]. Employing this method we prove the universality of anomalous conductivities such as the chiral magnetic and chiral separation conductiv- 
ities for theories holographically described by generic two-derivative gravity. This result establishes the holographic analog of the non-renormalization theorems found on the field theory side.

The paper is organized as follows. In section 2, we introduce the general holographic setting that we employ in this paper. Here we also fix the coefficients of the Chern-Simons terms by matching the anomaly equation on the field theory side.

In section 3 we introduce our ansatz for the fluctuation equations in order to calculate the anomalous conductivities. We study the near boundary and near horizon behavior of these fluctuations and show that the regularity of metric fluctuations near the horizon require vanishing of metric fluctuations there. In this section we also derive expressions for the conserved fluxes that correspond to these fluctuations.

In section 4 we finally evaluate the anomalous conductivities associated with background vector and axial sources and demonstrate universality of their values.

The final section discusses our results and our method and presents an outlook for further research.

\section{Action and equations of motion}

The model we work with in this paper is given by the following action

$$
\begin{aligned}
S=\frac{1}{16 \pi G} \int[R * 1 & -\frac{1}{2} \mathrm{~d} \phi \wedge * \mathrm{~d} \phi-\frac{\Psi(\phi)}{2} \mathrm{~d} \chi \wedge * \mathrm{~d} \chi-V(\phi) * 1-\frac{Z_{A}(\phi)}{2} F^{A} \wedge * F^{A} \\
& \left.-\frac{Z_{V}(\phi)}{2} F^{V} \wedge * F^{V}+\frac{\kappa}{3} A \wedge\left(F^{A} \wedge F^{A}+3 g F^{V} \wedge F^{V}\right)\right],
\end{aligned}
$$

with $F^{A}=\mathrm{d} A$ and $F^{V}=\mathrm{d} V$ are the field strengths for the bulk axial and vector fields dual to the axial and the vector currents in the dual field theory. The dilaton scalar field, $\phi$, and the axion one, $\chi$, will not play an explicit rôle in the solution of the fluctuations, even though we assume that they are non-trivial in the background. The main effect of the dilaton in this study comes from the dilatonic couplings $Z_{A, V}$. Here we assume that the one-forms $A$ and $V$ are normalized in such a way that

$$
\lim _{r \rightarrow \infty} Z_{A}(\phi)=\lim _{r \rightarrow \infty} Z_{V}(\phi)=1,
$$

with $r \rightarrow \infty$ corresponding to the boundary, where we assume the geometry becomes asymptotically $\mathrm{AdS}_{5}$. The normalization (2.2) can always be attained with a redefinition of the factors $\kappa$ and $g$ in the Chern-Simons (CS) terms.

The choice of CS terms in (2.1) corresponds to including a Bardeen counter-term in the boundary action [44] whose presence is required for an anomaly-free vector current. Indeed if we make a gauge transformation $V \rightarrow V+\mathrm{d} \zeta_{V}$ in (2.1) we obtain $\partial_{\mu} J^{\mu}=0$ for the vector current that is dual to the bulk gauge-field $V^{\mu}$.

The coefficients $\kappa$ and $g$ are not arbitrary, and their value can be found matching to the gauge anomaly of one left-handed and one right-handed fermion. With a gauge transformation $\delta_{\zeta_{A}}$ for the axial U(1) field $A$ we obtain

$$
\delta_{\zeta_{A}} S=\frac{\kappa}{48 \pi G}\left(F^{A} \wedge F^{A}+3 g F^{V} \wedge F^{V}\right)=-\partial_{\mu} J_{5}^{\mu} .
$$


On the other hand, in the presence of the Bardeen counter-term on the boundary theory, the correct anomaly equation for the axial current reads,

$$
\partial_{\mu} J_{5}^{\mu}=\frac{N_{c}}{12 \pi^{2}}\left(3 F^{V} \wedge F^{V}+F^{A} \wedge F^{A}\right) .
$$

Matching (2.3) with (2.4) we determine

$$
\kappa=-\frac{4 G N_{c}}{\pi}, \quad g=1 .
$$

From now on we set $g=1$.

The equations of motion from the variation of the action (2.1) read, for the scalars,

$$
\begin{aligned}
\mathrm{d}(\Psi * \mathrm{~d} \chi) & =0, \\
\mathrm{~d} * \mathrm{~d} \phi & =\partial_{\phi} V * 1+\frac{\partial_{\phi} Z_{A}}{2} F^{A} \wedge * F^{A}+\frac{\partial_{\phi} Z_{V}}{2} F^{V} \wedge * F^{V}+\frac{\partial_{\phi} \Psi}{2} \mathrm{~d} \chi \wedge * \mathrm{~d} \chi,
\end{aligned}
$$

for the gauge fields,

$$
\begin{aligned}
\mathrm{d}\left(Z_{A} * F^{A}-\kappa A \wedge F^{A}-\kappa V \wedge F^{V}\right) & =0 \\
\mathrm{~d}\left(Z_{V} * F^{V}-2 \kappa A \wedge F^{V}\right) & =0
\end{aligned}
$$

and for the metric

$$
\begin{aligned}
R_{\mu \nu}= & \frac{1}{2} \partial_{\mu} \phi \partial_{\nu} \phi+\frac{\Psi}{2} \partial_{\mu} \chi \partial_{\nu} \chi+\frac{V}{3} g_{\mu \nu}+\frac{Z_{A}}{2}\left(F_{\mu \rho}^{A} F_{\nu}^{A^{\rho}}-\frac{1}{6} g_{\mu \nu} F_{\rho \sigma}^{A} F^{A, \rho \sigma}\right) \\
& +\frac{Z_{V}}{2}\left(F_{\mu \rho}^{V} F_{\nu}^{V}-\frac{1}{6} g_{\mu \nu} F_{\rho \sigma}^{V} F^{V, \rho \sigma}\right) .
\end{aligned}
$$

We assume a static, translational and rotational invariant background given by the following configuration

$$
\begin{aligned}
\mathrm{d} s^{2} & =-g_{t t}(r) \mathrm{d} t^{2}+g_{x x}(r) \mathrm{d} \vec{x}^{2}+g_{r r}(r) \mathrm{d} r^{2}, \\
A & =A_{t}(r) \mathrm{d} t, \quad V=V_{t}(r) \mathrm{d} t, \quad \phi(r), \quad \chi(r) .
\end{aligned}
$$

Notice that $g_{t t}$ is a positive-definite function, and that AdS-RN with two charges falls into this general ansatz for the specific values $Z_{A}=Z_{V}=1, V(\phi)=-20, \chi=\phi=0$. If we further require $V_{t}=0$ we recover the case studied in section 3.2 of [52].

Near the boundary, as $r \rightarrow \infty$, we require that the solution becomes asymptotically $\mathrm{AdS}_{5}$

$$
g_{t t} \sim r^{2}+\cdots, \quad g_{x x} \sim r^{2}+\cdots, \quad g_{r r} \sim r^{-2}+\cdots,
$$

with the remaining functions asymptote to constants: $A_{t} \sim A_{t}^{\infty}+\cdots$, etc, ellipsis indicating the subleading terms.

In the deep interior of the background we require existence of a non-extremal horizon such that near the horizon, $r=r_{h}$, we have

$$
g_{t t} \sim t_{h}\left(r-r_{h}\right)+\cdots, \quad g_{x x} \sim x_{h}+\cdots, \quad g_{r r} \sim \frac{\rho_{h}}{r-r_{h}}+\cdots,
$$

whereas the remaining background functions again tend to constants: $A_{t} \sim A_{t}^{h}+\cdots$, etc. 
Before analysing the fluctuations of the system it is useful to express the temporal components of the gauge fields, $A_{t}$ and $V_{t}$, in terms of constants of motion. To this end let us first define

$$
\begin{aligned}
& J_{5}^{\mu \nu}=-\sqrt{-g} Z_{A}(\phi) F^{A, \mu \nu}+\frac{\kappa}{2} \epsilon^{\mu \nu \alpha \rho \sigma}\left(A_{\alpha} F_{\rho \sigma}^{A}+a_{\alpha} F_{\rho \sigma}^{V}\right) \\
& J^{\mu \nu}=-\sqrt{-g} Z_{V}(\phi) F^{V, \mu \nu}+\kappa \epsilon^{\mu \nu \alpha \rho \sigma} A_{\alpha} F_{\rho \sigma}^{V}
\end{aligned}
$$

for which the equations of motion (2.8) and (2.9) simply read

$$
\partial_{\mu} J_{5}^{\mu \nu}=0, \quad \partial_{\mu} J^{\mu \nu}=0
$$

For the background (2.11) the only non-vanishing components are

$$
J_{5, b g}^{r t}=\sqrt{\frac{g_{x x}^{3}}{g_{t t} g_{r r}}} Z_{A}(\phi) A_{t}^{\prime}, \quad J_{b g}^{r t}=\sqrt{\frac{g_{x x}^{3}}{g_{t t} g_{r r}}} Z_{V}(\phi) V_{t}^{\prime},
$$

which, upon use of equations of motion (2.8) are found to be constants

$$
J_{5, b g}^{r t}=Q_{5}, \quad J_{b g}^{r t}=Q
$$

\section{Fluctuations}

In this section we consider fluctuations on the background described above, with the purpose of determining anomalous transport coefficients. As shown for example in [44], it is sufficient to restrict ourselves to the following set of fluctuations with vanishing frequency and with a non-trivial spatial momentum that can be taken in the y-direction with no loss of generality:

$$
\begin{aligned}
\delta A & =\delta A_{x}(y, r) \mathrm{d} x+\delta A_{z}(y, r) \mathrm{d} z \\
\delta V & =\delta V_{x}(y, r) \mathrm{d} x+\delta V_{z}(y, r) \mathrm{d} z \\
\delta \mathrm{d} s^{2} & =2 \delta g_{t x}(y, r) \mathrm{d} t \mathrm{~d} x+2 \delta g_{t z}(y, r) \mathrm{d} t \mathrm{~d} z
\end{aligned}
$$

These fluctuations correspond to the vectorial sector preserving rotations in the $x-z$ plane. As a result they do not couple to fluctuations of other components of the metric or the gauge fields, nor to the scalars.

At this point we employ the method in [53] to introduce sources explicitly in these fluctuations. We turn them on for the gauge fields only as follows:

$$
\begin{aligned}
\delta A_{x}(y, r) & =-B_{z}^{5} y+\alpha_{x}(r), & & \delta A_{z}(y, r)=B_{x}^{5} y+\alpha_{z}(r), \\
\delta V_{x}(y, r) & =-B_{z} y+\beta_{x}(r), & & \delta V_{z}(y, r)=B_{x} y+\beta_{z}(r), \\
\delta g_{t x}(y, r) & =g_{x x} \gamma_{x}(r), & & \delta g_{t z}(y, r)=g_{x x} \gamma_{z}(r) .
\end{aligned}
$$

Here sources correspond to the (axio-)magnetic fields $B_{a}$ and $B_{a}^{5}$, with $a=\{x, z\}$. We thus require $\alpha_{a}$ and $\gamma_{a}$ correspond to normalizable deformations of the fields, that describe the response to the sources $B_{a}$ and $B_{a}^{5}$. Since the gauge fields and the graviton are 
massless, the holographic correspondence implies that we must have the following leading near boundary behavior: $\alpha_{a} \sim r^{-2}, \beta_{a} \sim r^{-2}$ and $\gamma_{a} \sim r^{-4}$.

To check this asymptotic behavior let us begin analyzing the equations of motion for the gauge fields fluctuations. With the definitions (2.15) and (2.16) these equations are

$$
\partial_{r} \delta J_{5}^{r a}+\partial_{y} \delta J_{5}^{y a}=0, \quad \partial_{r} \delta J^{r a}+\partial_{y} \delta J^{y a}=0,
$$

where $\delta J^{\mu a}$ and $\delta J_{5}^{\mu a}$ are the part of (2.16) and (2.15) linear in fluctuations on top of our background ansatz (2.11).

It turns out that the derivatives with respect to the spatial coordinate $y$ can be expressed as radial derivatives

$$
\partial_{y} \delta J_{5}^{y a}=-\left(\kappa A_{t}^{\prime} B_{b}^{5}+\kappa V_{t}^{\prime} B_{b}\right) \delta^{a b}, \quad \partial_{y} \delta J^{y a}=-2 \kappa V_{t}^{\prime} B_{b}^{5} \delta^{a b},
$$

with $\delta^{a b}$ Kronecker's delta. Therefore the equations of motion for the fluctuations of the gauge fields read

$$
\partial_{r} \tilde{J}_{5}^{a}=\partial_{r} \tilde{J}^{a}=0
$$

where we have defined

$$
\begin{aligned}
\tilde{J}_{5}^{a} & \equiv-\left(2 \kappa A_{t} B_{b}^{5}+2 \kappa V_{t} B_{b}+Q_{5} \gamma_{b}+\sqrt{\frac{g_{t t} g_{x x}}{g_{r r}}} Z_{A}(\phi) \alpha_{b}^{\prime}\right) \delta^{a b}, \\
\tilde{J}^{a} & \equiv-\left(2 \kappa A_{t} B_{b}+2 \kappa V_{t} B_{b}^{5}+Q \gamma_{b}+\sqrt{\frac{g_{t t} g_{x x}}{g_{r r}}} Z_{V}(\phi) \beta_{b}^{\prime}\right) \delta^{a b} .
\end{aligned}
$$

The quantities $\tilde{J}_{5}^{a}$ and $\tilde{J}^{a}$ are conserved on-shell along the radial direction. In particular they help determine the leading behavior of the $\alpha_{a}$ and $\beta_{a}$ fluctuations near the boundary. Provided $\gamma_{a} \sim r^{-4}$ we obtain

$$
\begin{aligned}
& \alpha_{a}(r) \simeq \frac{\tilde{J}_{5}^{b} \delta_{a b}+2 \kappa\left(A_{t}^{\infty} B_{a}^{5}+V_{t}^{\infty} B_{a}\right)}{2 r^{2}}+\cdots, \\
& \beta_{a}(r) \simeq \frac{\tilde{J}^{b} \delta_{a b}+2 \kappa\left(A_{t}^{\infty} B_{a}+V_{t}^{\infty} B_{a}^{5}\right)}{2 r^{2}}+\cdots,
\end{aligned}
$$

as $r \rightarrow \infty$. Notice the shift with respect to the conserved quantities $\tilde{J}_{5}^{a}$ in the numerator.

We must now prove that consistently $\gamma_{a} \sim r^{-4}$ near the boundary. This is straightforward once we realize that the equations of motion for the fluctuations $\gamma_{a}$ are simply

$$
\partial_{r} \tilde{K}_{a}=0
$$

where we have used the background equations of motion and defined two more constants of motion

$$
\tilde{K}_{a} \equiv \sqrt{\frac{g_{x x}^{5}}{g_{t t} g_{r r}}} \gamma_{a}^{\prime}+Q_{5} \alpha_{a}+Q \beta_{a} .
$$

Using the boundary behavior of the background solutions above we indeed find that

$$
\gamma_{\mu}(r) \simeq-\frac{\tilde{K}_{\mu}}{4 r^{4}}+\cdots \quad r \rightarrow \infty
$$


Let us now turn to the behavior of the fields near the horizon. With first present the general form of the Ricci scalar for the background perturbed by the fluctuations of the metric (3.4):

$$
R=R_{b g}+\frac{\mathcal{S}^{a} \gamma_{a}+\mathcal{S}^{a b} \gamma_{a} \gamma_{b}}{g_{t t}}
$$

Here $\mathcal{S}^{a}$ and $\mathcal{S}_{a b}$ are some radial functions that are regular at the horizon, and $R_{b g}$ is the Ricci scalar of the background solution. We observe a curvature singularity at the horizon unless $\gamma_{a}$ vanishes there, which we assume in the following. We will not need explicit expressions for $\gamma_{a}$ in what follows.

Requiring this near-horizon behavior in (3.10), (3.11) and (3.15) we find that the leading behavior of $\alpha_{a}$ is such that the fluctuations of the gauge fields indeed become constants at the horizon.

With these two results in hand one can finally determine the value of the constants of motion $\tilde{J}^{a}$ and $\tilde{J}_{5}^{a}$ at the horizon

$$
\begin{gathered}
\tilde{J}_{5}^{a}=-2 \kappa\left(A_{t}^{h} B_{b}^{5}+V_{t}^{h} B_{b}\right) \delta^{a b}, \\
\tilde{J}^{a}=-2 \kappa\left(A_{t}^{h} B_{b}+V_{t}^{h} B_{b}^{5}\right) \delta^{a b} .
\end{gathered}
$$

Having determined the behavior of the fields near the horizon and near the boundary, we specify completely the fluctuations $\alpha_{a}, \beta_{a}$ and $\gamma_{a}$. In the next section we see how this is sufficient to obtain the one-point functions associated to these fluctuations.

\section{Chiral magnetic and separation effects}

Equipped with the expressions (3.18) and (3.19) we can write the one-point functions corresponding to the expectation values of the axial and vector currents. This is given by the normalizable mode of the $\alpha_{a}$ in equation (3.12) and $\beta_{a}$ in equation (3.13), with fluctuations appropriately normalized. From action (2.1) the final result reads ${ }^{3}$

$$
\begin{aligned}
& \left\langle J_{5}^{a}\right\rangle=\frac{-\kappa}{8 \pi G}\left[\left(A_{t}^{\infty}-A_{t}^{h}\right) B_{b}^{5}+\left(V_{t}^{\infty}-V_{t}^{h}\right) B_{b}\right] \delta^{a b}=\frac{-\kappa}{8 \pi G}\left(\mu_{5} B_{b}^{5}+\mu B_{b}\right) \delta^{a b}, \\
& \left\langle J^{a}\right\rangle=\frac{-\kappa}{8 \pi G}\left[\left(A_{t}^{\infty}-A_{t}^{h}\right) B_{b}+\left(V_{t}^{\infty}-V_{t}^{h}\right) B_{b}^{5}\right] \delta^{a b}=\frac{-\kappa}{8 \pi G}\left(\mu_{5} B_{b}+\mu B_{b}^{5}\right) \delta^{a b},
\end{aligned}
$$

with $A_{t}^{\infty}-A_{t}^{h}=\mu_{5}$ and $V_{t}^{\infty}-V_{t}^{h}=\mu$ the associated chemical potentials. Substituting the value for $\kappa$ in (2.5) we obtain the one-point functions

$$
\begin{aligned}
\left\langle J_{5}^{a}\right\rangle & =\frac{N_{c}}{2 \pi^{2}}\left(\mu_{5} B_{b}^{5}+\mu B_{b}\right) \delta^{a b}, \\
\left\langle J^{a}\right\rangle & =\frac{N_{c}}{2 \pi^{2}}\left(\mu_{5} B_{b}+\mu B_{b}^{5}\right) \delta^{a b} .
\end{aligned}
$$

\footnotetext{
${ }^{3}$ Strictly speaking one should choose the gauge $A_{t}^{\infty}=V_{t}^{\infty}=0$ to avoid complications in the calculation by a redefinition of (4.1), see [54]. We discuss this point further in the Discussion section below.
} 
From these expressions we define the chiral conductivities as the derivatives of the one-point function with respect to each of the magnetic fields. We obtain

$$
\begin{aligned}
& \left\langle J^{a}\right\rangle=\sigma_{\mathrm{VV}} B^{a}+\sigma_{\mathrm{VA}} B^{5 a}, \\
& \left\langle J_{5}^{a}\right\rangle=\sigma_{\mathrm{AA}} B^{5 a}+\sigma_{\mathrm{AV}} B^{a},
\end{aligned}
$$

with

$$
\sigma_{\mathrm{VV}}=\sigma_{\mathrm{AA}}=\frac{N_{c}}{2 \pi^{2}} \mu_{5}, \quad \sigma_{\mathrm{AV}}=\sigma_{\mathrm{VA}}=\frac{N_{c}}{2 \pi^{2}} \mu
$$

In particular, for vanishing the axial magnetic, we obtain the desired chiral magnetic effect

$$
\left\langle J^{\nu}\right\rangle=\sigma_{\mathrm{VV}} B^{\nu}=\frac{N_{c}}{2 \pi^{2}} \mu_{5} B^{\nu}
$$

and chiral separation effect

$$
\left\langle J_{5}^{\nu}\right\rangle=\sigma_{\mathrm{AV}} B^{\nu}=\frac{N_{c}}{2 \pi^{2}} \mu B^{\nu} .
$$

We emphasize that our results (4.8) and (4.9) follows directly from the horizon universality of the fluctuations and, as such, they are valid in a generic class of theories where the gravitational description follows from the generic action (2.1).

\section{Discussion}

In this paper we demonstrated that the anomalous conductivity describing the chiral magnetic effect acquires a universal value for a generic holographic model. Our result is independent of the details of the background solution. As mentioned in the Introduction, this fact was already shown on the field theory side with a variety of different methods including the Ward identities and new non-renormalization theorems, hydrodynamics and effective field theories. Our calculation provides yet another, independent demonstration of this universal behavior, thus fills the gap on the dual gravitational side. It is reassuring that there exist a holographic analog to this field theory non-renormalization theorem, and it indeed follows from horizon universality, as one would expect.

The only requirement that we need in our calculation, besides the general form of the action, is a rather physical one: that the curvature scalar is finite at the horizon. This allowed us to express the conserved quantities (3.10), (3.11) and (3.15) in terms of horizon quantities, which recombined in a neat way with UV data in the solution for the fluctuations near the boundary to produce the chemical potentials of the theory in the final result.

We left out the calculation of the chiral vortical effect in our analysis. This can be studied by adding a gravitational Chern-Simons term to the action (2.1) of the form

$$
S \rightarrow S+\frac{\lambda}{16 \pi G} \int A \wedge \operatorname{tr}(\mathcal{R} \wedge \mathcal{R})
$$

with $\mathcal{R}^{\mu}{ }_{\nu}=R^{\mu}{ }_{\nu \rho \sigma} \mathrm{d} x^{\rho} \wedge \mathrm{d} x^{\sigma}$ the curvature tensor. One also needs to add a new boundary term to the action to make the variational problem well posed. This additional term 
produces an extra contribution $\lambda \operatorname{tr}(\mathcal{R} \wedge \mathcal{R})$ in the equation of motion (2.8) and a similar contribution in (2.10). For the fluctuations considered in this paper the former term vanishes, implying that our results for the anomalous conductivities in (4.7) are not affected by the presence of this additional contribution in the action. We plan to study all anomalous transport coefficients including the chiral vortical effect in future. This will provide a generalization of the results in section 4 of [52], for a gravity theory coupled to scalar matter.

We should also comment on a technical issue that was alluded to in the footnote on page 8. Our result should of course be independent of the choice of gauge for the bulk gauge fields. However, there is a subtlety [54] arising from two different methods to realize the chemical potentials $\mu$ and $\mu_{5}$ on gravity side. In the first method, that we employed here, one chooses a vanishing boundary value for the gauge fields. In this method the calculation goes straightforwardly with the definition (4.1). One may also choose a gauge where the gauge fields vanish on the horizon and asymptote to finite values on the boundary. In this case however there is another contribution to the boundary chiral current (4.1) that arises from the finite Chern-Simons current that should be added to the consistent current to obtain the covariant current. To establish gauge equivalence with the previous method one has to include a spurious boundary axion [54]. In this paper we chose to work with the first method, i.e. the formalism B in the nomenclature of [54].

As is common with general calculations showing a robust result, the setup we have considered in this paper may help identify ways to model setups where the chiral magnetic conductivity differs from the universal result found here. One such possibility follows by preventing the equation of motion for the axial gauge field fluctuation to have a constant of motion, (3.9). This situation is realized for example by a Stückelberg type action, where the kinetic term for the axion in (2.1) now reads

$$
(16 \pi G) S_{\chi \chi}=-\int \frac{\Psi(\phi)}{2}\left(\mathrm{~d} \chi-m_{A} A\right) \wedge *\left(\mathrm{~d} \chi-m_{A} A\right)
$$

with $m_{A}$ the mass of the axial field. Indeed this mechanism was proposed as a holographic dual to anomalous theories with type II anomalies - that is, with a gluonic contribution to the anomaly equation $\left(a_{3} \neq 0\right.$ in equation (1.2) $)$ - in [48] and [55]. The bulk axion is dual to a theta-term in the field theory action. The idea is that coupling $\chi$ to the axial field induces a non-trivial expectation value for $\langle\operatorname{Tr} G \wedge G\rangle$ in (1.2). The anomaly term associated with this expectation value, $a_{3}$, then induces a modification of the result for the anomalous transport coefficients $[48,55]$. In holographic constructions of QCD $m_{A}$ is usually proportional to the number of flavors in the theory, and one needs a calculation in the Veneziano limit to see the effect of this term [56].

There are various possible extensions of our work. First of all, as already mentioned above, the extension of the holographic non-renormalization found here to the case of chiral vortical conductivity would be very interesting. Secondly, we wonder if our universal result for the chiral conductivities survive the higher derivative corrections in gravity. It is well-known that one generates corrections to the shear viscosity in presence of higher derivative corrections [57], but the notion of horizon universality continues to hold. In the 
case of anomalous transport we also expect horizon universality to determine the values of conductivities exactly, also in presence of higher derivative terms. Whether the actual value changes or not remains to be seen. In this context one should note a physical distinction between viscosity and anomalous conductivities, namely the former is dissipative and the latter is not. It is conceivable therefore that the result we found here may be robust against higher derivative corrections as one expects from the non-renormalization of these conductivities on the field theory side. Thirdly, one may wonder if the kind of universality we find here extends to finite frequency and momenta. It would be also very interesting to predict such universal behavior in the momenta dependence of the anomalous conductivities through a holographic study. Finally, it should be possible to extend our calculations to the case of holographic superfluids whose broken $U(1)$ is anomalous [58].

\section{Acknowledgments}

We thank Francisco Pena-Benitez, Aron Jansen and especially Aristomenis Donos for interesting discussions and useful remarks. JT is grateful to the Mainz Institute for Theoretical Physics (MITP) for its hospitality and partial support during the initial stage of this work.

This work is part of the D-ITP consortium, a program of the Netherlands Organisation for Scientific Research (NWO) that is funded by the Dutch Ministry of Education, Culture and Science (OCW).

JT is supported by grants 2014-SGR-1474, MEC FPA2010-20807-C02-01, MEC FPA2010-20807-C02-02, ERC Starting Grant HoloLHC-306605 and by the Juan de la Cierva program of the Spanish Ministry of Economy.

Open Access. This article is distributed under the terms of the Creative Commons Attribution License (CC-BY 4.0), which permits any use, distribution and reproduction in any medium, provided the original author(s) and source are credited.

\section{References}

[1] D.E. Kharzeev, L.D. McLerran and H.J. Warringa, The effects of topological charge change in heavy ion collisions: 'Event by event P and CP-violation', Nucl. Phys. A 803 (2008) 227 [arXiv:0711.0950] [INSPIRE].

[2] K. Fukushima, D.E. Kharzeev and H.J. Warringa, The chiral magnetic effect, Phys. Rev. D 78 (2008) 074033 [arXiv:0808.3382] [InSPIRE].

[3] V. Skokov, A. Yu. Illarionov and V. Toneev, Estimate of the magnetic field strength in heavy-ion collisions, Int. J. Mod. Phys. A 24 (2009) 5925 [arXiv:0907.1396] [InSPIRE].

[4] K. Tuchin, Synchrotron radiation by fast fermions in heavy-ion collisions, Phys. Rev. C 82 (2010) 034904 [Erratum ibid. C 83 (2011) 039903] [arXiv:1006. 3051] [INSPIRE].

[5] V. Voronyuk, V.D. Toneev, W. Cassing, E.L. Bratkovskaya, V.P. Konchakovski and S.A. Voloshin, (Electro-)magnetic field evolution in relativistic heavy-ion collisions, Phys. Rev. C 83 (2011) 054911 [arXiv:1103.4239] [inSPIRE]. 
[6] W.-T. Deng and X.-G. Huang, Event-by-event generation of electromagnetic fields in heavy-ion collisions, Phys. Rev. C 85 (2012) 044907 [arXiv: 1201.5108] [INSPIRE].

[7] K. Tuchin, Particle production in strong electromagnetic fields in relativistic heavy-ion collisions, Adv. High Energy Phys. 2013 (2013) 490495 [arXiv:1301.0099] [INSPIRE].

[8] L. McLerran and V. Skokov, Comments about the electromagnetic field in heavy-ion collisions, Nucl. Phys. A 929 (2014) 184 [arXiv:1305.0774] [inSPIRE].

[9] U. Gürsoy, D. Kharzeev and K. Rajagopal, Magnetohydrodynamics, charged currents and directed flow in heavy ion collisions, Phys. Rev. C 89 (2014) 054905 [arXiv:1401.3805] [INSPIRE].

[10] STAR collaboration, B.I. Abelev et al., Azimuthal charged-particle correlations and possible local strong parity violation, Phys. Rev. Lett. 103 (2009) 251601 [arXiv:0909.1739] [INSPIRE].

[11] STAR collaboration, S.A. Voloshin, Probe for the strong parity violation effects at RHIC with three particle correlations, Indian J. Phys. 85 (2011) 1103 [arXiv:0806.0029] [INSPIRE].

[12] C. Zhang et al., Observation of the Adler-Bell-Jackiw chiral anomaly in a Weyl semimetal, arXiv:1503.02630 [inSPIRE].

[13] S.Y. Xu et al., Discovery of a Weyl fermion semimetal and topological Fermi arcs, Science 349 (2015) 613 [arXiv:1502.03807].

[14] D.E. Kharzeev and H.-U. Yee, Anomaly induced chiral magnetic current in a Weyl semimetal: chiral electronics, Phys. Rev. B 88 (2013) 115119 [arXiv:1207.0477] [INSPIRE].

[15] G. Basar, D.E. Kharzeev and H.-U. Yee, Triangle anomaly in Weyl semimetals, Phys. Rev. B 89 (2014) 035142 [arXiv: 1305.6338] [INSPIRE].

[16] K. Landsteiner, Anomalous transport of Weyl fermions in Weyl semimetals, Phys. Rev. B 89 (2014) 075124 [arXiv: 1306.4932] [INSPIRE].

[17] S.L. Adler, Axial vector vertex in spinor electrodynamics, Phys. Rev. 177 (1969) 2426 [INSPIRE].

[18] J.S. Bell and R. Jackiw, A PCAC puzzle: $\pi^{0} \rightarrow \gamma \gamma$ in the $\sigma$-model, Nuovo Cim. A 60 (1969) 47 [inSPIRE].

[19] V.A. Kuzmin, V.A. Rubakov and M.E. Shaposhnikov, On the anomalous electroweak baryon number nonconservation in the early universe, Phys. Lett. B 155 (1985) 36 [INSPIRE].

[20] P.B. Arnold and L.D. McLerran, The sphaleron strikes back, Phys. Rev. D 37 (1988) 1020 [INSPIRE].

[21] P.B. Arnold and L.D. McLerran, Sphalerons, small fluctuations and baryon number violation in electroweak theory, Phys. Rev. D 36 (1987) 581 [INSPIRE].

[22] M.A. Metlitski and A.R. Zhitnitsky, Anomalous axion interactions and topological currents in dense matter, Phys. Rev. D 72 (2005) 045011 [hep-ph/0505072] [INSPIRE].

[23] G.M. Newman and D.T. Son, Response of strongly-interacting matter to magnetic field: some exact results, Phys. Rev. D 73 (2006) 045006 [hep-ph/0510049] [INSPIRE].

[24] D.E. Kharzeev and H.J. Warringa, Chiral magnetic conductivity, Phys. Rev. D 80 (2009) 034028 [arXiv:0907.5007] [INSPIRE]. 
[25] K. Jensen, Triangle anomalies, thermodynamics and hydrodynamics, Phys. Rev. D 85 (2012) 125017 [arXiv: 1203.3599] [InSPIRE].

[26] N. Banerjee et al., Constraints on fluid dynamics from equilibrium partition functions, JHEP 09 (2012) 046 [arXiv:1203.3544] [INSPIRE].

[27] V.P. Nair, R. Ray and S. Roy, Fluids, anomalies and the chiral magnetic effect: a group-theoretic formulation, Phys. Rev. D 86 (2012) 025012 [arXiv:1112.4022] [INSPIRE].

[28] A.V. Sadofyev and M.V. Isachenkov, The chiral magnetic effect in hydrodynamical approach, Phys. Lett. B 697 (2011) 404 [arXiv:1010.1550] [InSPIRE].

[29] A.V. Sadofyev, V.I. Shevchenko and V.I. Zakharov, Notes on chiral hydrodynamics within effective theory approach, Phys. Rev. D 83 (2011) 105025 [arXiv: 1012.1958] [INSPIRE].

[30] K. Jensen, P. Kovtun and A. Ritz, Chiral conductivities and effective field theory, JHEP 10 (2013) 186 [arXiv:1307.3234] [INSPIRE].

[31] N. Banerjee, S. Dutta, S. Jain, R. Loganayagam and T. Sharma, Constraints on anomalous fluid in arbitrary dimensions, JHEP 03 (2013) 048 [arXiv: 1206.6499] [INSPIRE].

[32] S. Golkar and D.T. Son, (Non)-renormalization of the chiral vortical effect coefficient, JHEP 02 (2015) 169 [arXiv:1207.5806] [INSPIRE].

[33] D.-F. Hou, H. Liu and H.-c. Ren, A possible higher order correction to the vortical conductivity in a gauge field plasma, Phys. Rev. D 86 (2012) 121703 [arXiv:1210.0969] [INSPIRE].

[34] M. Knecht, S. Peris, M. Perrottet and E. de Rafael, New nonrenormalization theorems for anomalous three point functions, JHEP 03 (2004) 035 [hep-ph/0311100] [INSPIRE].

[35] P.V. Buividovich, Anomalous transport with overlap fermions, Nucl. Phys. A 925 (2014) 218 [arXiv: 1312.1843] [INSPIRE].

[36] D.T. Son and P. Surowka, Hydrodynamics with triangle anomalies, Phys. Rev. Lett. 103 (2009) 191601 [arXiv:0906.5044] [INSPIRE].

[37] Y. Neiman and Y. Oz, Relativistic hydrodynamics with general anomalous charges, JHEP 03 (2011) 023 [arXiv: 1011.5107] [INSPIRE].

[38] K. Jensen, R. Loganayagam and A. Yarom, Thermodynamics, gravitational anomalies and cones, JHEP 02 (2013) 088 [arXiv:1207.5824] [INSPIRE].

[39] J.M. Maldacena, The large- $N$ limit of superconformal field theories and supergravity, Int. J. Theor. Phys. 38 (1999) 1113 [Adv. Theor. Math. Phys. 2 (1998) 231] [hep-th/9711200] [INSPIRE].

[40] S.S. Gubser, I.R. Klebanov and A.M. Polyakov, Gauge theory correlators from noncritical string theory, Phys. Lett. B 428 (1998) 105 [hep-th/9802109] [INSPIRE].

[41] E. Witten, Anti-de Sitter space and holography, Adv. Theor. Math. Phys. 2 (1998) 253 [hep-th/9802150] [INSPIRE].

[42] J. Erdmenger, M. Haack, M. Kaminski and A. Yarom, Fluid dynamics of R-charged black holes, JHEP 01 (2009) 055 [arXiv:0809.2488] [INSPIRE].

[43] N. Banerjee, J. Bhattacharya, S. Bhattacharyya, S. Dutta, R. Loganayagam and P. Surowka, Hydrodynamics from charged black branes, JHEP 01 (2011) 094 [arXiv:0809.2596] [INSPIRE]. 
[44] A. Gynther, K. Landsteiner, F. Pena-Benitez and A. Rebhan, Holographic anomalous conductivities and the chiral magnetic effect, JHEP 02 (2011) 110 [arXiv:1005.2587] [INSPIRE].

[45] I. Amado, K. Landsteiner and F. Pena-Benitez, Anomalous transport coefficients from Kubo formulas in holography, JHEP 05 (2011) 081 [arXiv:1102.4577] [INSPIRE].

[46] K. Landsteiner, E. Megias and F. Pena-Benitez, Gravitational anomaly and transport, Phys. Rev. Lett. 107 (2011) 021601 [arXiv:1103.5006] [InSPIRE].

[47] K. Landsteiner, E. Megias, L. Melgar and F. Pena-Benitez, Holographic gravitational anomaly and chiral vortical effect, JHEP 09 (2011) 121 [arXiv:1107.0368] [INSPIRE].

[48] U. Gürsoy and A. Jansen, (Non)renormalization of anomalous conductivities and holography, JHEP 10 (2014) 92 [arXiv:1407.3282] [INSPIRE].

[49] G. Policastro, D.T. Son and A.O. Starinets, The shear viscosity of strongly coupled $N=4$ supersymmetric Yang-Mills plasma, Phys. Rev. Lett. 87 (2001) 081601 [hep-th/0104066] [INSPIRE].

[50] A. Buchel and J.T. Liu, Universality of the shear viscosity in supergravity, Phys. Rev. Lett. 93 (2004) 090602 [hep-th/0311175] [InSPIRE].

[51] N. Iqbal and H. Liu, Universality of the hydrodynamic limit in AdS/CFT and the membrane paradigm, Phys. Rev. D 79 (2009) 025023 [arXiv:0809.3808] [InSPIRE].

[52] K. Landsteiner and L. Melgar, Holographic flow of anomalous transport coefficients, JHEP 10 (2012) 131 [arXiv:1206.4440] [INSPIRE].

[53] A. Donos and J.P. Gauntlett, The thermoelectric properties of inhomogeneous holographic lattices, JHEP 01 (2015) 035 [arXiv:1409.6875] [INSPIRE].

[54] K. Landsteiner, E. Megias and F. Pena-Benitez, Anomalous transport from Kubo formulae, Lect. Notes Phys. 871 (2013) 433 [arXiv:1207.5808] [INSPIRE].

[55] A. Jimenez-Alba, K. Landsteiner and L. Melgar, Anomalous magnetoresponse and the Stückelberg axion in holography, Phys. Rev. D 90 (2014) 126004 [arXiv:1407.8162] [INSPIRE].

[56] U. Gursoy, A. Jansen and J. Tarrio, Anomalous conductivities and holography in the Veneziano limit.

[57] M. Brigante, H. Liu, R.C. Myers, S. Shenker and S. Yaida, Viscosity bound violation in higher derivative gravity, Phys. Rev. D 77 (2008) 126006 [arXiv:0712.0805] [INSPIRE].

[58] I. Amado, N. Lisker and A. Yarom, Universal chiral conductivities for low temperature holographic superfluids, JHEP 06 (2014) 084 [arXiv: 1401.5795] [INSPIRE]. 